\title{
Prognostic value of left atrial size and function in adults with tetralogy of Fallot
}

\author{
Vivan J.M. Baggen ${ }^{\mathrm{a}, \mathrm{b}}$, Anne-Rose W. Schut ${ }^{\mathrm{a}}$, Judith A.A.E. Cuypers ${ }^{\mathrm{a}}$, Maarten Witsenburg ${ }^{\text {a }}$, Eric Boersma ${ }^{\mathrm{a}, \mathrm{b}, \mathrm{c}}$, \\ Annemien E. van den Bosch ${ }^{a}$, Jolien W. Roos-Hesselink ${ }^{\mathrm{a}, *}$ \\ a Department of Cardiology, Erasmus Medical Center, Rotterdam, The Netherlands \\ b Cardiovascular Research School COEUR, Rotterdam, The Netherlands \\ c Department of Clinical Epidemiology, Erasmus Medical Center, Rotterdam, The Netherlands
}

\section{A R T I C L E I N F O}

\section{Article history:}

Received 22 November 2016

Received in revised form 27 January 2017

Accepted 24 February 2017

Available online xxxx

\section{Keywords:}

Left atrium

Tetralogy of Fallot

Prognosis

Adverse events

\begin{abstract}
A B S T R A C T
Background: Left atrial (LA) size predicts cardiovascular outcome in chronic heart failure. Its prognostic value in adults with repaired tetralogy of Fallot (ToF) is unknown. This study therefore investigated the association of LA size and function with cardiovascular events in adults with ToF.

Methods: Clinically stable adults with ToF who visited the outpatient clinic between 2011 and 2013 underwent echocardiography and were prospectively followed for the occurrence of death, heart failure, hospitalizations, arrhythmia, thromboembolic events, and re-interventions. LA maximal, minimal and pre-A wave volume, area and length were measured on the apical four-chamber view. Total, passive and active emptying fractions were calculated.

Results: In total, 134 patients were included (median age 35 [IQR 29-45] years, 65\% male, 91\% NYHA I). Median follow-up was 40 [IQR 32-47] months. Patients with a dilated LA ( $\left.\geq 34 \mathrm{~mL} / \mathrm{m}^{2}, 43 \%\right)$ were at higher risk of cardiovascular events $(n=33$, adjusted HR 2.48 [1.09-5.62], $P=0.030)$. Analysis of LA volumes as continuous variables yielded similar conclusions. In addition, LA length (adjusted HR 2.49 [1.51-4.09], $P<0.001$ ), total emptying fraction (adjusted HR 0.96 [0.93-0.99], $P=0.008$ ), and active emptying fraction (adjusted HR 0.92 [0.87-0.96], $P=0.001$ ) were significantly associated with cardiovascular events. Standardized HRs indicated that LA length was the strongest prognostic marker. In addition, none of the patients with a normally sized LA died or developed heart failure.

Conclusions: LA size and function can provide relevant prognostic information in clinically stable adults with repaired ToF. Especially LA length may be a valuable additional tool in the risk stratification of these patients.
\end{abstract}

(c) 2017 Published by Elsevier Ireland Ltd.

\section{Introduction}

Tetralogy of Fallot (ToF) is the most common cyanotic heart defect $[1,2]$. Thanks to successful repair at young age, the survival of patients with ToF has tremendously improved with current survival rates over $90 \%$ up to 30 years after surgical repair [3]. Nevertheless, residual lesions are common. Especially older patients are at risk of complications such as heart failure, arrhythmia and early demise. Therefore, life-long follow-up is warranted. Identification of patients with a high risk of adverse cardiovascular events is essential, in order to optimize patient information, follow-up and therapeutic strategies [3,4].

Echocardiography is the cornerstone in the evaluation of cardiac function in patients with ToF. Right and left ventricular function are known as important prognostic markers in these patients [5]. Atrial function has been largely neglected so far. The left atrium (LA) has

* Corresponding author at: Erasmus University Medical Center, Department of Cardiology, Room Ba-583a, P.O. Box 2040, 3000 CA Rotterdam, The Netherlands.

E-mail address: j.roos@erasmusmc.nl (J.W. Roos-Hesselink). multiple functions: it modulates as a reservoir, a conduit and as a pump [6]. LA size can be reliably measured with two-dimensional echocardiography and reflects the average effect of left ventricle (LV) filling pressures over time. It is known as a useful marker for both chronicity and severity of LV diastolic dysfunction [7-9]. Larger LA volumes have been associated with an increased risk of adverse cardiovascular events in patients with chronic heart failure [10]. To the best of our knowledge, the prognostic value of LA size or function in patients with ToF has not been evaluated. Therefore, the aim of this study was to investigate the association of LA size and function with a composite endpoint of cardiovascular events in clinically stable adults with repaired ToF.

\section{Methods}

\subsection{Study design and population}

For the purpose of this study, all patients with repaired ToF were selected from a large prospective cohort of clinically stable adults with 
congenital heart disease. This cohort consisted of consecutive patients who routinely visited our adult congenital cardiology outpatient clinic and underwent echocardiography between April 2011 and April 2013. Patients with pulmonary valve atresia and ventricular septal defect and patients with isolated pulmonary stenosis were excluded. Other exclusion criteria were: age $<18$ years, pregnancy, incapability of understanding and signing informed consent, kidney failure (creatinine $>200 \mu \mathrm{mol} / \mathrm{L}$ ), or insufficient echocardiographic image quality. Image quality was defined as insufficient when the LA borders were not clearly visible and therefore could not be adequately traced in the apical fourchamber (A4C) view.

At the time of study inclusion, all patients underwent physical examination by a cardiologist, 12-lead ECG, venous blood sampling, and echocardiography. Patient demographics, medical history, medication use, symptoms and signs of heart failure (NYHA classification), results of physical examination, associated lesions, surgical characteristics, re-interventions, electrocardiography, echocardiography, laboratory results and events were collected using an electronic CRF-based online system (๔ 2004-2012 OpenClinica, LLC and collaborators). Details have been reported previously [11]. The institutional review board of the Erasmus MC approved the study protocol and all participants provided written informed consent.

\subsection{Echocardiography}

Two-dimensional transthoracic echocardiographic images were acquired using a commercially available ultrasound system iE33 (Philips Medical Systems, Best, the Netherlands) with a $1.5-\mathrm{MHz}$ transducer. Echocardiographic measurements were performed in agreement with the current recommendations of the American Society of Echocardiography and European Association of Cardiovascular Imaging [12]. LV end-diastolic and end systolic volumes were calculated using the biplane method of disks summation (modified Simpson's rule). LV systolic function was quantified with LV ejection fraction, and visually graded as normal or impaired. LV diastolic function was assessed using pulsed waved Doppler of the mitral valve inflow (E/Aratio and deceleration time) and septal tissue Doppler imaging (E' wave and E/E'-ratio). RV systolic function was quantified with RV fractional area change and tricuspid annular plane systolic excursion.

\subsection{Left atrial size and function}

LA volumetric measurements were performed on the A4C view in three different phases during one cardiac cycle. LA maximal and minimal volume were measured on the frames with respectively the largest and smallest atrial cavities on visual assessment. LA pre-A-wave volume was measured one frame before the atrial contraction started (also based on the movement of the mitral valve leaflets, and the onset of the P-wave on the ECG). Contours were manually traced, the atrial appendage and pulmonary veins were excluded, and at the mitral valve level the contour was closed with a straight line. For the calculation of volumes a single plane summation of disks method was used. In addition, left atrial maximal size was quantified using the LA anteroposterior dimension on the parasternal long axis view, the LA major axis on the $\mathrm{A} 4 \mathrm{C}$ view (LA length), and the LA maximal area on the A4C view (Supplemental File 1). All LA volumes were indexed by body surface area.

We separately considered LA reservoir, conduit and contractile function $[6,7,13]$. Reservoir function was quantified with LA total emptying volume (TEV), LA total emptying fraction (TEF) and the expansion index. TEV was calculated by LA maximal volume - LA minimal volume. TEF was calculated by (TEV/LA maximal volume) ${ }^{*} 100 \%$. Expansion index was defined as (TEV/LA minimal volume) ${ }^{*} 100 \%$. Conduit function was quantified with LA passive emptying volume (PEV) and LA passive emptying fraction (PEF). PEV was calculated by LA maximal volume - LA pre-A-wave volume. PEF was calculated by (PEV/LA maximal volume)
* $100 \%$. Contractile or active function was estimated with LA active emptying volume (AEV) and LA active emptying fraction (AEF). AEV was calculated by LA pre-A-wave volume - LA minimal volume. AEF was calculated by (AEV/LA pre-A-wave volume) $* 100 \%$.

One investigator (A.S.), who was blinded for clinical events and other patient data, performed all LA measurements. Reproducibility was assessed by blinded repeated analysis of 20 randomly selected patients by the first and a second observer (V.B.).

\subsection{Laboratory testing}

Peripheral venous blood samples were obtained from all patients after at least 30 minutes of rest. Serum NT-proBNP was measured for research purposes only in our clinical chemistry laboratory using a commercial electrochemiluminescence immunoassay (Roche Diagnostics, Rotkreuz, Switzerland). The limit of detection was $0.6 \mathrm{pmol} / \mathrm{L}$. The upper limit of normal for NT-proBNP was $14 \mathrm{pmol} / \mathrm{L}$ (approximately $125 \mathrm{pg} / \mathrm{mL}$ ), based on the recommended low cut-off for the diagnosis of heart failure in patients presenting with non-acute symptoms [14].

\subsection{Definition and assessment of events}

The primary endpoint was defined prior to the collection of data as a composite of all adverse cardiovascular events: all-cause mortality, heart failure (requiring initiation or change in heart failure medication, or requiring hospital admission), hospitalization for cardiac reasons, arrhythmia (symptomatic and recorded, or requiring treatment), thromboembolic events (ischemic cerebrovascular accident, pulmonary embolism or myocardial infarction), or cardiac re-interventions (surgical or percutaneous). A secondary composite endpoint of allcause mortality or heart failure was defined. All patients were prospectively followed for fatal and non-fatal events by a yearly clinical evaluation at our institution. During the follow-up, patients were treated according to the physician's discretion and the study protocol did allow for adjustment of cardiac medication. Where necessary, we retrieved information from electronic patient charts and correspondence with referring hospitals. Survival status of all patients was also checked in the Municipal Population Register. Events were adjudicated by two experienced investigators (V.B. and J.R.) without knowledge of LA measurements. The investigators had direct access to the patient clinical records and the events were independently reviewed by each investigator. For patients with multiple events, event-free survival was defined as the time from enrollment to the occurrence of the first event. Patients without any cardiovascular event were censored at the end of the follow-up duration (August 1, 2015).

\subsection{Statistical analysis}

Continuous normally distributed data were presented as mean \pm standard deviation (SD) and skewed data as median $\left[\mathrm{IQ}_{1}-\mathrm{IQ}_{3}\right]$. Categorical data were presented as frequencies and percentages. Following the guidelines, patients were stratified according to LA volume index $<34 \mathrm{~mL} / \mathrm{m}^{2}$ (normal LA) and $\geq 34 \mathrm{~mL} / \mathrm{m}^{2}$ (dilated LA) [12]. Continuous variables were compared between these two groups using the Student's $t$-test or the Mann-Whitney $U$ test, depending on the distribution of data. Categorical variables were compared using the Chi-squared test or Fisher's exact test when applicable. Cumulative endpoint free survival estimates and survival curves were derived by the KaplanMeier method; the log-rank test was used to compare groups. Cox proportional hazards regression was used to investigate the associations between LA measurements and endpoints. LA measurements were analyzed as continuous and as standardized variables (created by calculating z-scores). The association of LA measurements with the primary endpoint was adjusted for age and NYHA functional class, using a minimum of ten endpoints per degree of freedom to prevent overfitting. Inter- and intra-observer agreement of LA maximal volume, 
area and length were presented by Bland-Altman plots. Limits of agreement were defined as the mean difference of the two repeated measurements $\pm 1.96 \mathrm{SD}$. The coefficient of variation (COV) was defined as the SD of the differences of two measurements divided by the mean of two measurements * $100 \%$. Mean relative differences were calculated by dividing the difference of two measurements by the mean of two measurements ${ }^{*} 100 \%$. All data analysis was performed using IBM SPSS Statistics Version 21.0.0.1 (IBM Corp., Armonk, NY, USA). Two-sided $P$-values of $<0.05$ were considered statistically significant.

\section{Results}

\subsection{Baseline measurements}

Of the 179 patients that were included in the initial cohort, 31 patients with pulmonary valve atresia and 1 patient with isolated pulmonary stenosis were excluded. In addition, 13 patients were excluded because of insufficient image quality. These patients did not have significant or clinically relevant differences in age, NYHA classification, body mass index, use of cardiac medication or number of re-interventions compared to the 134 adult patients with repaired ToF who were included in this study. Median age at inclusion was 35 [2945 ] years and 87 patients (65\%) were male. Median age at initial repair was 3.3 [1.0-6.7] years and in 87 patients (67\%) a transannular patch was used. Baseline characteristics are further detailed in Table 1.

The LA was dilated $\left(\geq 34 \mathrm{~mL} / \mathrm{m}^{2}\right)$ in 58 patients (43\%). Clinical characteristics and results of electrocardiography and echocardiography are compared between patients with a normal and a dilated LA in Tables 1 and 2. Patients with a dilated LA were significantly older and were in a higher NYHA functional class. In addition, 22 patients (38\%) with a dilated LA used cardiac medication compared with 9 patients (12\%) with a normal LA $(P<0.001)$. The majority of patients were in sinus rhythm ( $\mathrm{n}=111,83 \%)$ and had a prolonged QRS interval $(\mathrm{n}=91$, $75 \%$ ). None of the patients fulfilled criteria of heart failure at baseline. Patients with a dilated LA had significantly larger LV volumes, impaired diastolic function (expressed by a lower E' wave and a higher E/E'-ratio), a larger RV end-diastolic annulus dimension and a higher NT-proBNP level. No significant differences in rhythm or valvular function were found between both subgroups.

\subsection{Follow-up}

At the end of the follow-up (August 1, 2015), survival status and detailed follow-up data were complete in 134 patients (100\%). During a median follow-up period of 40.4 [31.9-46.7] months, the primary endpoint occurred in 33 patients (25\%) and the secondary endpoint occurred in 8 patients (6\%). None of the patients with a normally sized

Table 1

Baseline characteristics of the study cohort.

\begin{tabular}{|c|c|c|c|c|c|}
\hline & \multirow[b]{2}{*}{ Valid cases, n (\%) } & \multirow[b]{2}{*}{ All patients } & \multicolumn{2}{|l|}{ LA volume index, $\mathrm{mL} / \mathrm{m}^{2}$} & \multirow[b]{2}{*}{$P$-value } \\
\hline & & & $<34$ (normal, $\mathrm{n}=76$ ) & $\geq 34$ (dilated, $\mathrm{n}=58)$ & \\
\hline \multicolumn{6}{|l|}{ Clinical characteristics } \\
\hline Age, years & $134(100)$ & 35 [29-45] & 31 [26-39] & 42 [34-49] & $<0.001$ \\
\hline Sex, male n (\%) & $134(100)$ & $87(65)$ & $49(65)$ & $38(66)$ & 0.900 \\
\hline NYHA class II-III, n (\%) & $134(100)$ & $12(9)$ & $3(4)$ & $9(16)$ & 0.020 \\
\hline Body mass index, $\mathrm{kg} / \mathrm{m}^{2}$ & $134(100)$ & $24 \pm 4$ & $24 \pm 4$ & $25 \pm 4$ & 0.046 \\
\hline Body surface area, $\mathrm{m}^{2}$ & $134(100)$ & $1.89 \pm 0.20$ & $1.89 \pm 0.22$ & $1.89 \pm 0.18$ & 0.918 \\
\hline Systolic blood pressure, $\mathrm{mmHg}$ & $131(98)$ & $126 \pm 17$ & $124 \pm 15$ & $127 \pm 19$ & 0.355 \\
\hline $\mathrm{O}_{2}$ saturation $<90 \%, \mathrm{n}(\%)$ & $126(94)$ & $1(1)$ & $1(1)$ & $0(0)$ & - \\
\hline Cardiac medication, n (\%) & $134(100)$ & $31(23)^{*}$ & $9(12)$ & $22(38)$ & $<0.001$ \\
\hline ACE-inhibitor & $134(100)$ & $10(8)$ & $4(5)$ & $6(10)$ & - \\
\hline Beta blocker & $134(100)$ & $17(13)$ & $3(4)$ & $14(24)$ & - \\
\hline Anti-arrhythmic & $134(100)$ & $10(8)$ & $2(3)$ & $8(14)$ & - \\
\hline Aspirin & $134(100)$ & $10(8)$ & $2(3)$ & $8(14)$ & - \\
\hline Anticoagulants & $134(100)$ & $16(12)$ & $1(1)$ & $15(26)$ & - \\
\hline Diuretics & $134(100)$ & $7(5)$ & $0(0)$ & $7(12)$ & - \\
\hline Associated lesions, $\mathrm{n}(\%)$ & $134(100)$ & $31(23)$ & $15(20)$ & $16(28)$ & 0.286 \\
\hline Atrial septal defect & $134(100)$ & $16(12)$ & $7(9)$ & $9(16)$ & - \\
\hline Patent ductus arteriosus & $133(99)$ & $11(8)$ & $7(9)$ & $4(7)$ & - \\
\hline Aortic valve stenosis & $134(100)$ & $8(6)$ & $4(5)$ & $5(7)$ & - \\
\hline \multicolumn{6}{|l|}{ Surgical characteristics } \\
\hline Age at repair, years & $134(100)$ & $3.3[1.0-6.7]$ & $1.6[0.8-4.1]$ & $5.8[2.2-9.7]$ & $<0.001$ \\
\hline Prior aortopulmonary shunt, n (\%) & $134(100)$ & $39(29)$ & $14(18)$ & $25(43)$ & 0.002 \\
\hline Blalock shunt & $134(100)$ & $20(15)$ & $5(7)$ & $15(26)$ & - \\
\hline Waterston shunt & $134(100)$ & $16(12)$ & $7(9)$ & $9(16)$ & - \\
\hline Other shunt & $134(100)$ & $3(2)$ & $2(2)$ & $1(2)$ & - \\
\hline Transannular patch used, n (\%) & $129(96)$ & $87(67)$ & $52(71)$ & $35(63)$ & 0.294 \\
\hline Re-intervention, n (\%) & $134(100)$ & $86(64)$ & $48(63)$ & $38(66)$ & 0.778 \\
\hline More than two re-interventions & $134(100)$ & $14(10)$ & $4(5)$ & $10(17)$ & 0.025 \\
\hline Surgical, n (\%) & $134(100)$ & $75(56)$ & $46(61)$ & $29(50)$ & 0.224 \\
\hline Pulmonary valve replacement & $134(100)$ & $67(50)$ & $42(55)$ & $25(43)$ & - \\
\hline Residual VSD repair & $134(100)$ & $19(14)$ & $8(11)$ & $11(19)$ & - \\
\hline Catheter-based, n (\%) & $134(100)$ & $19(14)$ & $11(15)$ & $8(14)$ & 0.911 \\
\hline Pulmonary valve balloon dilatation & $134(100)$ & $11(8)$ & $7(9)$ & $4(7)$ & - \\
\hline Pulmonary valve replacement & $134(100)$ & $4(3)$ & $2(3)$ & $2(3)$ & - \\
\hline Ablation & $134(100)$ & $4(3)$ & $2(3)$ & $2(3)$ & - \\
\hline Other, n (\%) & $134(100)$ & $22(16)$ & $9(12)$ & $13(22)$ & 0.102 \\
\hline
\end{tabular}

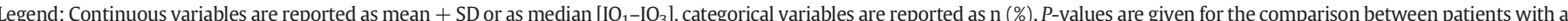

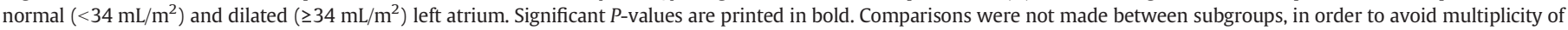
comparisons. *In 13 patients the cardiac medication was changed during the follow-up.

Abbreviations: ACE, angiotensin converting enzyme; LA, left atrial; NYHA, New York Heart Association; VSD, ventricular septal defect. 
Table 2

Baseline electrocardiography, echocardiography and laboratory results of the study cohort.

\begin{tabular}{|c|c|c|c|c|c|}
\hline & \multirow[b]{2}{*}{ Valid cases, n (\%) } & \multirow[b]{2}{*}{ All patients } & \multicolumn{2}{|l|}{ LA volume index, $\mathrm{mL} / \mathrm{m}^{2}$} & \multirow[b]{2}{*}{$P$-value } \\
\hline & & & $<34($ normal, $\mathrm{n}=76)$ & $\geq 34$ (dilated, $\mathrm{n}=58$ ) & \\
\hline \multicolumn{6}{|l|}{ Electrocardiography } \\
\hline Heart rate, beats/min & $134(100)$ & $75 \pm 13$ & $76 \pm 14$ & $73 \pm 13$ & 0.298 \\
\hline \multicolumn{6}{|l|}{ Rhythm, n (\%) } \\
\hline Sinus rhythm & $134(100)$ & $111(83)$ & $67(88)$ & $44(76)$ & 0.061 \\
\hline Pacemaker rhythm & $134(100)$ & $12(9)$ & $4(5)$ & $8(14)$ & 0.087 \\
\hline Other* & $134(100)$ & $11(8)$ & $5(7)$ & $6(10)$ & 0.531 \\
\hline QRS duration, ms $\dagger$ & $122(91)$ & 144 [120-169] & $139[119-164]$ & $158[122-170]$ & 0.286 \\
\hline QRS duration $>120 \mathrm{~ms}, \mathrm{n}(\%)^{*}$ & $122(91)$ & $91(75)$ & $52(72)$ & $39(78)$ & 0.471 \\
\hline RBBB & $91(100)$ & $82(90)$ & $50(96)$ & $32(82)$ & - \\
\hline LBBB & $91(100)$ & $1(1)$ & $0(0)$ & $1(3)$ & - \\
\hline Other & $91(100)$ & $8(9)$ & $2(4)$ & $6(15)$ & - \\
\hline PR interval, if sinus rhythm, ms & $111(83)$ & $165[146-180]$ & $163[146-173]$ & $173[148-187]$ & 0.053 \\
\hline \multicolumn{6}{|l|}{ Echocardiography } \\
\hline LV end diastolic volume, $\mathrm{mL} / \mathrm{m}^{2}$ & $122(91)$ & $56[49-66]$ & $54[45-64]$ & $59[51-71]$ & 0.027 \\
\hline LV end systolic volume, $\mathrm{mL} / \mathrm{m}^{2}$ & $121(90)$ & $25[20-32]$ & $24[21-30]$ & $28[21-34]$ & 0.048 \\
\hline LV ejection fraction, \% & $122(91)$ & $53 \pm 8$ & $54 \pm 5$ & $53 \pm 10$ & 0.446 \\
\hline Normal LV function, $\mathrm{n}(\%)$ & $134(100)$ & $58(43)$ & $33(43)$ & $25(43)$ & 0.971 \\
\hline E/A-ratio & $128(95)$ & $1.5[1.1-2.1]$ & $1.5[1.1-2.1]$ & $1.4[1.1-2.0]$ & 0.338 \\
\hline E' wave, $\mathrm{cm} / \mathrm{s}$ & $129(96)$ & $7.7[6.2-9.2]$ & $8.1[6.6-9.6]$ & $7.0[5.8-8.8]$ & 0.023 \\
\hline E/E'-ratio & $127(95)$ & $10.0[8.1-13.6]$ & $8.9[7.7-11.9]$ & $11.3[8.4-15.8]$ & 0.005 \\
\hline Deceleration time, ms & $124(93)$ & $192[158-235]$ & $192[158-237]$ & $192[159-235]$ & 0.835 \\
\hline RV end-diastolic annulus, mm & $119(89)$ & $45.7 \pm 7.9$ & $43.7 \pm 7.7$ & $48.4 \pm 7.4$ & 0.001 \\
\hline TAPSE, mm & $124(93)$ & $17.4 \pm 4.5$ & $17.1 \pm 4.4$ & $17.8 \pm 4.8$ & 0.432 \\
\hline RV fractional area change, \% & $105(78)$ & $41 \pm 9$ & $41 \pm 9$ & $42 \pm 9$ & 0.443 \\
\hline \multicolumn{6}{|l|}{ Valvular function } \\
\hline AV peak velocity, m/s & $117(87)$ & $1.07[0.94-1.21]$ & $1.05[0.91-1.16]$ & $1.12[0.96-1.24]$ & 0.078 \\
\hline AR $\geq$ moderate, $\mathrm{n}(\%)$ & $89(66)$ & $3(3)$ & $0(0)$ & $3(7)$ & 0.101 \\
\hline PV peak velocity, m/s & $131(98)$ & $2.20 \pm 0.75$ & $2.24 \pm 0.73$ & $2.15 \pm 0.78$ & 0.519 \\
\hline $\mathrm{PR} \geq$ moderate, $\mathrm{n}(\%)$ & $124(93)$ & $47(38)$ & $25(37)$ & $22(39)$ & 0.773 \\
\hline TR $\geq$ moderate, $\mathrm{n}(\%)$ & $124(93)$ & $11(9)$ & $5(7)$ & $6(11)$ & 0.536 \\
\hline TR peak velocity, m/s & $107(80)$ & $2.8 \pm 0.5$ & $2.8 \pm 0.5$ & $2.9 \pm 0.6$ & 0.276 \\
\hline MR $\geq$ moderate, $\mathrm{n}(\%)$ & $82(61)$ & $1(1)$ & $0(0)$ & $1(2)$ & 1.000 \\
\hline \multicolumn{6}{|l|}{ Laboratory results } \\
\hline Hemoglobin, mmol/L & $93(69)$ & $9.2 \pm 0.9$ & $9.3 \pm 0.8$ & $9.0 \pm 1.0$ & 0.181 \\
\hline Creatinine, $\mu \mathrm{mol} / \mathrm{L}$ & $134(100)$ & $78.4 \pm 19.5$ & $76.7 \pm 13.7$ & $80.7 \pm 25.2$ & 0.237 \\
\hline NT-proBNP, pmol/L & $133(99)$ & $14.5[6.4-27.4]$ & $9.3[4.9-17.0]$ & $23.4[14.6-42.4]$ & $<0.001$ \\
\hline
\end{tabular}

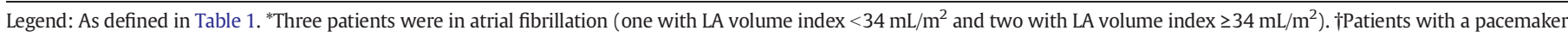
rhythm were excluded.

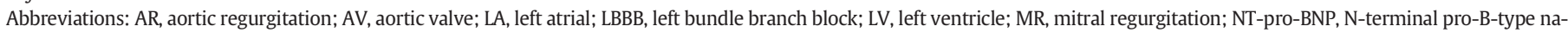

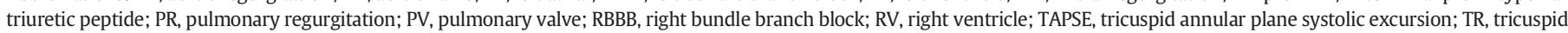
regurgitation.

LA died or developed heart failure. Kaplan-Meier estimates of event-free survival (the primary endpoint) were $88 \%$ at year one, $83 \%$ at year two and $77 \%$ at year three. Heart-failure-free survival (the secondary

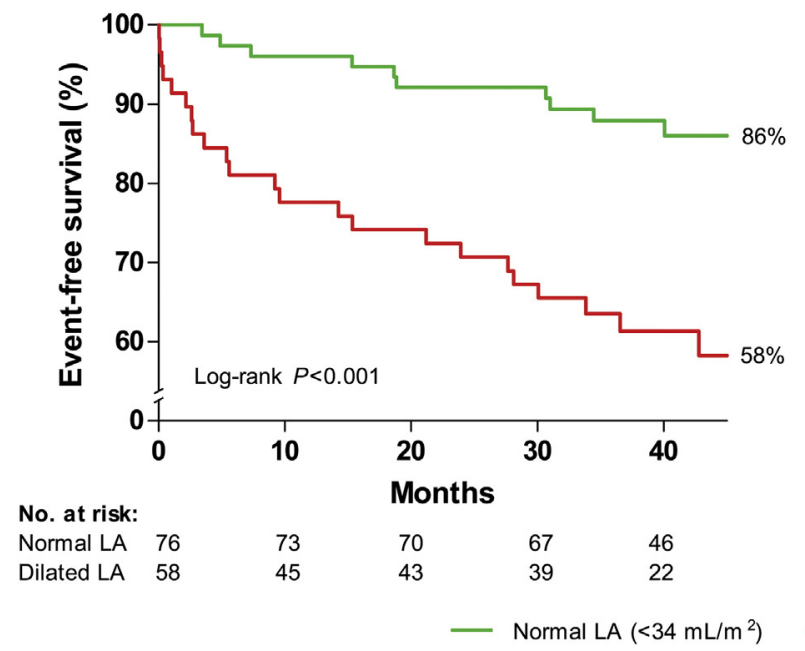

endpoint) was $96 \%$ at year one, $96 \%$ at year two and $94 \%$ at year three. All components of the primary and secondary endpoint are separately displayed in Supplemental File 2 for exploratory purposes.

Fig. 1. Cardiovascular event-free survival and heart failure-free survival, stratified according to LA maximal volume index $\left(<34 \mathrm{~mL} / \mathrm{m}^{2}\right.$ and $\left.\geq 34 \mathrm{~mL} / \mathrm{m}^{2}\right)$. 


\subsection{Prognostic value of left atrial measurements}

In Fig. 1, Kaplan-Meier curves show the proportion of patients free of the primary and secondary endpoint for patients with a normal and a dilated LA. In patients with a normally sized LA, the cumulative proportion of death and heart failure was $0 \%$. In Table 3 , the association between all LA measurements and the primary endpoint is shown. Except for the anteroposterior dimension on the parasternal long-axis view, all other LA size measurements (length, area, and volume) were significantly associated with the primary endpoint, also when adjusted for age and NYHA functional class. The standardized adjusted hazard ratios (HRs) show that among the various LA size indices, LA length was most strongly associated with the primary endpoint (HR 2.05 [95\% CI 1.38-3.02], $P<0.001$ ). In addition, a lower reservoir function (TEF) and a lower contractile function (AEF) were significantly associated with the primary endpoint, whereas a lower conduit function (PEF) was not.

\subsection{Reproducibility of left atrial measurements}

Bland-Altman plots of the intra- and inter-observer agreement of LA maximal volume, area and length are presented in Supplemental File 3. Mean differences of the intra-observer measurements were $-0.3 \pm$ $0.9 \mathrm{~mL} / \mathrm{m}^{2}(\mathrm{COV}=2.5), 0.0 \pm 0.4 \mathrm{~cm}^{2} / \mathrm{m}^{2}(\mathrm{COV}=3.4)$ and $0.0 \pm$ $0.2 \mathrm{~cm} / \mathrm{m}^{2}(\mathrm{COV}=5.7)$, and mean relative differences were $-0.7 \pm$ $2.8 \%,-0.2 \pm 3.5 \%$ and $-0.4 \pm 5.7 \%$ for LA maximal volume, area, and length, respectively. Mean differences of the inter-observer measurements were $-0.3 \pm 5.2 \mathrm{~mL} / \mathrm{m}^{2}(\mathrm{COV}=14.2), 0.0 \pm 1.0 \mathrm{~cm}^{2} / \mathrm{m}^{2}$ $(\mathrm{COV}=8.4)$ and $0.0 \pm 0.2 \mathrm{~cm} / \mathrm{m}^{2}(\mathrm{COV}=5.8)$, and mean relative differences were $-0.5 \pm 15.1 \%,-0.2 \pm 8.9 \%$ and $-0.8 \pm 5.6 \%$ for LA maximal volume, area, and length, respectively.

\section{Discussion}

To the best of our knowledge, this is the first study that evaluates the prognostic value of LA size and function in ToF patients. In our cohort of clinically stable adults with repaired ToF, the LA was enlarged in $43 \%$ of patients. Larger LA size, lower LA reservoir function, and lower LA contractile function were significantly associated with the primary endpoint of any cardiovascular event. None of the patients with a normally sized LA died or developed heart failure. Among the different indices of LA size, LA length was most strongly associated with adverse cardiovascular events, and had the best reproducibility.

\subsection{Diastolic dysfunction}

A potential association of LA size and clinical outcomes in patients with repaired ToF has been suggested earlier, but this association has not further been described [15]. However from other cardiac conditions, as well as in the general population, it is known that LA size is a marker of LV diastolic dysfunction and that it is associated with adverse clinical outcome [7-10,16,17]. In our cohort, patients with a dilated LA were significantly older, had larger LV volumes, and indeed had an impaired diastolic function. Of note, the accuracy of LV diastolic parameters such as the E' wave is questioned in patients with ToF because of the presence of the interventricular septal patch. Evaluation of LA size may therefore be a more reliable alternative, or valuable addition to the set of LV diastolic function parameters.

Cardiac magnetic resonance studies have evaluated the extent of ventricular fibrosis in patients with ToF using late gadolinium enhancement $[18,19]$. In this relatively young patient group, more prominent LV regional and diffuse myocardial fibrosis was observed, which was related to both abnormal systolic and diastolic function. Increased myocardial fibrosis may explain why LV diastolic dysfunction and accompanied LA enlargement already develops at a relatively young age in patients with ToF $[18,19]$. However, the normal aging process is also likely to play a role in the development of diastolic dysfunction, which was reflected in our cohort by increased LA volumes in older patients.

In this study, a decreased LA active emptying fraction was associated with cardiovascular events, whereas the passive emptying fraction was

The association of left atrial size and function with the primary composite endpoint.

\begin{tabular}{|c|c|c|c|c|c|c|c|c|c|c|}
\hline & \multirow[b]{2}{*}{$\begin{array}{l}\text { Valid cases, } \\
\mathrm{n}(\%)\end{array}$} & \multirow[b]{2}{*}{ Mean \pm SD } & \multirow[b]{2}{*}{$\begin{array}{l}\text { Crude HR } \\
(95 \% \mathrm{CI})\end{array}$} & \multirow[b]{2}{*}{$P$-value } & \multirow[b]{2}{*}{$\begin{array}{l}\text { Adjusted HR } \\
(95 \% \mathrm{CI})^{*}\end{array}$} & \multirow[b]{2}{*}{$P$-value } & \multicolumn{4}{|c|}{ Standardized values } \\
\hline & & & & & & & $\begin{array}{l}\text { Crude HR } \\
(95 \% \mathrm{CI})\end{array}$ & $P$-value & $\begin{array}{l}\text { Adjusted HR } \\
(95 \% \mathrm{CI})^{*}\end{array}$ & $P$-value \\
\hline \multicolumn{11}{|l|}{ LA size } \\
\hline AP dimension, $\mathrm{cm} \dagger$ & $124(93)$ & $3.9 \pm 0.8$ & $2.59(1.80-3.73)$ & $<\mathbf{0 . 0 0 1}$ & $2.00(1.25-3.21)$ & 0.004 & $2.16(1.61-2.90)$ & $<\mathbf{0 . 0 0 1}$ & $1.75(1.20-2.57)$ & 0.004 \\
\hline AP dimension, $\mathrm{cm} / \mathrm{m}^{2} \dagger$ & $124(93)$ & $2.1 \pm 0.4$ & $3.85(1.97-7.53)$ & $<0.001$ & $2.10(0.86-5.12)$ & 0.102 & $1.82(1.35-2.45)$ & $<0.001$ & $1.39(0.94-2.07)$ & 0.102 \\
\hline Length, $\mathrm{cm} \dagger$ & $134(100)$ & $5.5 \pm 0.8$ & $3.38(2.10-5.42)$ & $<\mathbf{0 . 0 0 1}$ & $2.49(1.51-4.09)$ & $<0.001$ & $2.60(1.79-3.77)$ & $<0.001$ & $2.05(1.38-3.02)$ & $<0.001$ \\
\hline Length, $\mathrm{cm} / \mathrm{m}^{2} \dagger$ & $134(100)$ & $2.9 \pm 0.5$ & $4.47(2.06-9.72)$ & $<0.001$ & $2.87(1.26-6.54)$ & 0.012 & $2.00(1.39-2.86)$ & $<0.001$ & $1.63(1.11-2.38)$ & 0.012 \\
\hline Area, $\mathrm{cm}^{2} / \mathrm{m}^{2}$ & $134(100)$ & $11.5 \pm 2.6$ & $1.35(1.21-1.50)$ & $<0.001$ & $1.24(1.07-1.43)$ & 0.004 & $2.16(1.65-2.84)$ & $<0.001$ & $1.73(1.20-2.51)$ & 0.004 \\
\hline Maximal volume $\geq 34 \mathrm{~mL} / \mathrm{m}^{2}$ & $134(100)$ & - & $3.71(1.76-7.80)$ & 0.001 & $2.48(1.09-5.62)$ & 0.030 & - & - & - & - \\
\hline Maximal volume, $\mathrm{mL} / \mathrm{m}^{2}$ & $134(100)$ & $36.0 \pm 12.6$ & $1.06(1.04-1.08)$ & $<\mathbf{0 . 0 0 1}$ & $1.04(1.01-1.07)$ & 0.007 & $2.06(1.61-2.64)$ & $<0.001$ & $1.63(1.14-2.33)$ & 0.007 \\
\hline Minimal volume, $\mathrm{mL} / \mathrm{m}^{2} \ddagger$ & $130(97)$ & $20.1 \pm 10.0$ & $1.06(1.04-1.09)$ & $<0.001$ & $1.04(1.01-1.07)$ & 0.006 & $1.85(1.51-2.26)$ & $<0.001$ & $1.51(1.13-2.03)$ & 0.006 \\
\hline Pre-A-wave volume, $\mathrm{mL} / \mathrm{m}^{2} \ddagger$ & $129(96)$ & $25.6 \pm 9.8$ & $1.07(1.04-1.10)$ & $<0.001$ & $1.06(1.02-1.10)$ & 0.003 & $1.93(1.52-2.45)$ & $<0.001$ & $1.75(1.21-2.51)$ & 0.003 \\
\hline \multicolumn{11}{|l|}{ LA function } \\
\hline \multicolumn{11}{|l|}{ Reservoir function } \\
\hline $\mathrm{TEV}, \mathrm{mL} / \mathrm{m}^{2}$ & $130(97)$ & $16.1 \pm 5.2$ & $1.06(1.00-1.13)$ & 0.070 & $1.02(0.95-1.09)$ & 0.603 & $1.36(0.98-1.89)$ & 0.070 & $1.10(0.78-1.54)$ & 0.603 \\
\hline $\mathrm{TEF}, \%$ & $130(97)$ & $45.5 \pm 9.2$ & $0.94(0.91-0.97)$ & $<\mathbf{0 . 0 0 1}$ & $0.96(0.93-0.99)$ & 0.008 & $0.57(0.43-0.74)$ & $<0.001$ & $0.67(0.50-0.90)$ & 0.008 \\
\hline Expansion index, \% & $130(97)$ & $88.4 \pm 31.1$ & $0.98(0.96-0.99)$ & 0.000 & $0.98(0.97-1.00)$ & 0.007 & $0.47(0.32-0.70)$ & $<0.001$ & $0.59(0.39-0.86)$ & 0.007 \\
\hline \multicolumn{11}{|l|}{ Conduit function } \\
\hline $\mathrm{PEV}, \mathrm{mL} / \mathrm{m}^{2}$ & $129(96)$ & $9.7 \pm 4.0$ & $1.07(0.98-1.16)$ & 0.127 & $1.05(0.97-1.14)$ & 0.234 & $1.30(0.93-1.82)$ & 0.127 & $1.22(0.88-1.71)$ & 0.234 \\
\hline PEF, \% & $129(96)$ & $27.9 \pm 9.0$ & $0.97(0.93-1.01)$ & 0.087 & $0.99(0.95-1.03)$ & 0.482 & $0.74(0.53-1.05)$ & 0.087 & $0.88(0.61-1.26)$ & 0.482 \\
\hline \multicolumn{11}{|l|}{ Contractile function } \\
\hline $\mathrm{AEV}, \mathrm{mL} / \mathrm{m}^{2}$ & $127(95)$ & $6.4 \pm 2.8$ & $1.11(0.99-1.25)$ & 0.071 & $0.99(0.86-1.14)$ & 0.883 & $1.35(0.97-1.88)$ & 0.071 & $0.97(0.65-1.44)$ & 0.883 \\
\hline $\mathrm{AEF}, \%$ & 127 (95) & $25.1 \pm 7.6$ & $0.94(0.90-0.98)$ & 0.008 & $0.92(0.87-0.96)$ & 0.001 & $0.62(0.44-0.88)$ & 0.008 & $0.51(0.34-0.76)$ & 0.001 \\
\hline
\end{tabular}

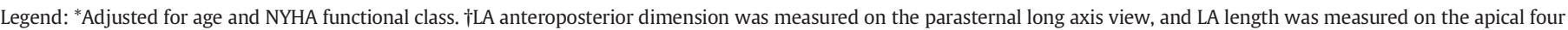

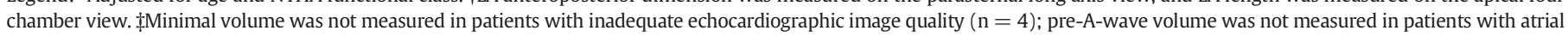

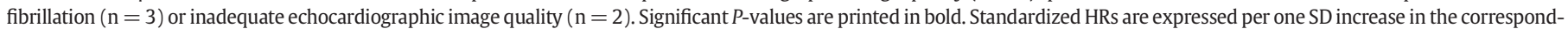
ing LA measurement.

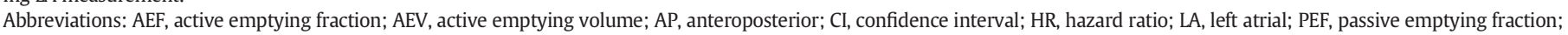
PEV, passive emptying volume; TEF, total emptying fraction; TEV, total emptying volume. 
not. This association has also been described in the general population, in chronic hypertension, and in ischemic heart disease [13,20]. When LV diastolic dysfunction progresses, adequate diastolic filling of the LV becomes more dependent on the contractile function of the LA [20]. This might explain why the contribution of the LA active emptying fraction seems to be of clinical importance in patients with ToF, in contrast to the passive emptying fraction. This is in line with a previous study in asymptomatic young patients after ToF repair, in which earlier and increased LA active contraction was suggested to indicate adaptive compensatory mechanisms to overcome latent and asymptomatic altered LV performance [21].

\subsection{Left atrial enlargement and clinical worsening}

RV dilatation and dysfunction have always been the major concern in patients with repaired ToF [1]. More recently, LV systolic dysfunction has also shown to predict adverse outcomes in ToF patients [5,22,23]. Several studies showed an association between RV and LV systolic dysfunction in ToF patients, suggesting an unfavorable ventricular interaction $[5,24,25]$. For instance, RV dilatation - secondary to RV volume overload - may shift the interventricular septum to the left, changing LV geometry, mechanics and filling [26]. More specific, speckletracking echocardiography studies have suggested that RV dilatation impedes LV apical rotation in patients with repaired ToF. The subsequent reduced LV twist resulted in global LV systolic dysfunction [24,25,27]. Interestingly, the abnormal systolic LV twist was also related to LV diastolic dysfunction in these patients [24]. Diastolic dysfunction can lead to an increase in LA pressure, reflected by LA enlargement. The resulting increase in pulmonary artery pressure may augment pulmonary regurgitation, which is a common problem in ToF patients [1]. The subsequent RV dilatation may create one of the vicious circles that can tilt a stable ToF patient towards progressive clinical deterioration.

Atrial fibrillation and mitral regurgitation are also known to influence the LA size [28]. In patients with ToF, atrial fibrillation is an important cause of morbidity and poor clinical outcome [15,28,29]. LA dilatation in patients with mitral regurgitation has been described as a compensatory mechanism and predictor of cardiovascular events [28, 29]. Both conditions could in theory explain the association of LA enlargement and clinical deterioration. However, in our cohort they do not seem to play a major role, since we found no significant differences in rhythm and heart rate between patients with a normal and a dilated LA, only three patients were in atrial fibrillation, and only one patient had moderate mitral regurgitation.

\subsection{Comparison of left atrial size measurements}

According to the guidelines, the most reliable and accurate method to estimate LA size and function is provided by LA volume or by multiple linear dimensions. A single linear measurement of the LA may underestimate LA size, considering the asymmetrical shape and the possibility of foreshortening $[8,12,16,30]$. In order to compare the prognostic value of the different measurements of LA size (volume, area, length, and anteroposterior dimension), we evaluated each measurement as a standardized variable, which allows comparison of the HRs across all variables. Interestingly, a single measurement of LA length appeared to be more predictive than LA volume, reflected by a higher standardized $H R$. This could be explained by the observation that LA dilatation mainly develops in the craniocaudal direction. Anatomic factors such as a dilated right ventricle, the sternum and the spine may limit LA dilatation in the anteroposterior direction [31]. As a result, LA length possibly provides a better reflection of LA enlargement. In addition, LA length showed to be the most reproducible measurement, reflected by a smaller interobserver variation than the LA area or volume measurement.

\subsection{Clinical implications}

In patients with ToF, accurate risk prediction is of paramount importance in order to optimize individual follow-up strategies, pharmacological treatment and timing of interventions [1]. This study shows that one-dimensional LA length is a simple and reliable measurement, which is associated with cardiovascular events in clinically stable adults with repaired ToF. This measurement comes at no extra costs, as it is quick and can be performed on the A4C view of routinely acquired echocardiographic images. Although other imaging techniques such as CT, CMR or 3D echocardiography could provide a more accurate estimation of LA size, this simple linear dimension can be easily implemented in day-to-day clinical practice. Patients with LA dilatation may require more vigilant follow-up, initiation or expansion of medical therapy, or earlier timing of a re-intervention. Importantly, LA size seems to have a very high negative predictive value for death and heart failure, which makes it particularly valuable as a screening tool. Patients with a normally sized LA may be reassured, and may need less frequent evaluation at the outpatient clinic.

\subsection{Study limitations}

The most important limitation of this study is that LA volumes were measured using a single plane approach of the A4C only, because the two-chamber views were of insufficient quality in the majority of patients. Because of this limitation, the LA volumes measured in our study could be slightly underestimated [31]. Second, due to the limited number of events, we could only adjust for the variables age and NYHA functional class, which may have resulted in residual confounding. Age at initial repair and prior palliative shunts were associated with LA dilatation, and may also play a crucial role. Larger studies are warranted to investigate the prognostic value of LA size and function in addition to multiple clinical, echocardiographic and biochemical markers.

\section{Conclusions}

In this cohort of clinically stable adults with repaired ToF, the LA was enlarged in a substantial number of patients. LA size and function, measured on routine echocardiographic examination, were significantly associated with cardiovascular events. A normally sized LA could accurately rule out the risk of death and heart failure with a high negative predictive value. LA length was most strongly associated with cardiovascular events, and had the best reproducibility. This free, quick and simple one-dimensional echocardiographic measurement may therefore be a valuable additional tool in the management and risk stratification of adults with repaired ToF.

\section{Conflicts of interest}

The authors report no relationships that could be construed as a conflict of interest.

\section{Acknowledgment}

This study was supported by a grant from the Dutch Heart Foundation, Den Haag, the Netherlands (grant number 2015T029).

\section{Appendix A. Supplementary data}

Supplementary data to this article can be found online at http://dx. doi.org/10.1016/j.ijcard.2017.02.153.

\section{References}

[1] C. Apitz, G.D. Webb, A.N. Redington, Tetralogy of Fallot, Lancet 374 (2009) 1462-1471. 
[2] D. van der Linde, E.E. Konings, M.A. Slager, et al., Birth prevalence of congenital heart disease worldwide: a systematic review and meta-analysis, J. Am. Coll. Cardiol. 58 (2011) 2241-2247.

[3] J.A. Cuypers, M.E. Menting, E.E. Konings, et al., Unnatural history of tetralogy of Fallot: prospective follow-up of 40 years after surgical correction, Circulation 130 (2014) 1944-1953.

[4] E.J. Hickey, G. Veldtman, T.J. Bradley, et al., Late risk of outcomes for adults with repaired tetralogy of Fallot from an inception cohort spanning four decades, Eur. J. Cardiothorac. Surg. 35 (2009) 156-164 (discussion 164).

[5] T. Geva, B.M. Sandweiss, K. Gauvreau, J.E. Lock, A.J. Powell, Factors associated with impaired clinical status in long-term survivors of tetralogy of Fallot repair evaluated by magnetic resonance imaging. J. Am. Coll. Cardiol. 43 (2004) 1068-1074.

[6] A.C. Boyd, N.B. Schiller, D. Leung, D.L. Ross, L. Thomas, Atrial dilation and altered function are mediated by age and diastolic function but not before the eighth decade, JACC Cardiovasc. Imaging 4 (2011) 234-242.

[7] D.Y. Leung, A. Boyd, A.A. Ng, C. Chi, L. Thomas, Echocardiographic evaluation of left atrial size and function: current understanding, pathophysiologic correlates, and prognostic implications, Am. Heart J. 156 (2008) 1056-1064.

[8] To AC, S.D. Flamm, T.H. Marwick, A.L. Klein, Clinical utility of multimodality LA imaging: assessment of size, function, and structure, JACC Cardiovasc. Imaging 4 (2011) 788-798.

[9] A.M. Pritchett, D.W. Mahoney, S.J. Jacobsen, R.J. Rodeheffer, B.L. Karon, M.M. Redfield, Diastolic dysfunction and left atrial volume: a population-based study, J. Am. Coll. Cardiol. 45 (2005) 87-92.

[10] V. Melenovsky, S.J. Hwang, M.M. Redfield, R. Zakeri, G. Lin, B.A. Borlaug, Left atrial remodeling and function in advanced heart failure with preserved or reduced ejection fraction, Circ. Heart Fail. 8 (2015) 295-303.

[11] J.A. Eindhoven, A.E. van den Bosch, T.P. Ruys, et al., N-terminal pro-B-type natriuretic peptide and its relationship with cardiac function in adults with congenital heart disease, J. Am. Coll. Cardiol. 62 (2013) 1203-1212.

[12] R.M. Lang, L.P. Badano, V. Mor-Avi, et al., Recommendations for cardiac chamber quantification by echocardiography in adults: an update from the American Society of Echocardiography and the European Association of Cardiovascular Imaging, J. Am. Soc. Echocardiogr. 28 (2015) 1-39 (e14).

[13] B.D. Hoit, Left atrial size and function: role in prognosis, J. Am. Coll. Cardiol. 63 (2014) 493-505.

[14] J.J. McMurray, S. Adamopoulos, S.D. Anker, et al., ESC guidelines for the diagnosis and treatment of acute and chronic heart failure 2012: the task force for the diagnosis and treatment of acute and chronic heart failure 2012 of the European Society of Cardiology. Developed in collaboration with the Heart Failure Association (HFA) of the ESC, Eur. Heart J. 33 (2012) 1787-1847.

[15] J. Roos-Hesselink, M.G. Perlroth, J. McGhie, S. Spitaels, Atrial arrhythmias in adults after repair of tetralogy of Fallot. Correlations with clinical, exercise, and echocardiographic findings, Circulation 91 (1995) 2214-2219.

[16] W.P. Abhayaratna, J.B. Seward, C.P. Appleton, et al., Left atrial size: physiologic determinants and clinical applications, J. Am. Coll. Cardiol. 47 (2006) 2357-2363.
[17] S.F. Nagueh, C.P. Appleton, T.C. Gillebert, et al., Recommendations for the evaluation of left ventricular diastolic function by echocardiography, Eur. J. Echocardiogr. 10 (2009) 165-193.

[18] S.V. Babu-Narayan, P.J. Kilner, W. Li, et al., Ventricular fibrosis suggested by cardiovascular magnetic resonance in adults with repaired tetralogy of fallot and its relationship to adverse markers of clinical outcome, Circulation 113 (2006) 405-413.

[19] M.F. Kozak, A. Redington, S.J. Yoo, M. Seed, A. Greiser, L. Grosse-Wortmann, Diffuse myocardial fibrosis following tetralogy of Fallot repair: a T1 mapping cardiac magnetic resonance study, Pediatr. Radiol. 44 (2014) 403-409.

[20] M. Kaminski, K. Steel, M. Jerosch-Herold, et al., Strong cardiovascular prognostic implication of quantitative left atrial contractile function assessed by cardiac magnetic resonance imaging in patients with chronic hypertension, J. Cardiovasc. Magn. Reson. 13 (2011) 42.

[21] K. Koenigstein, T. Raedle-Hurst, M. Hosse, M. Hauser, H. Abdul-Khaliq, Altered diastolic left atrial and ventricular performance in asymptomatic patients after repair of tetralogy of Fallot, Pediatr. Cardiol. 34 (2013) 948-953.

[22] A. Ghai, C. Silversides, L. Harris, G.D. Webb, S.C. Siu, J. Therrien, Left ventricular dysfunction is a risk factor for sudden cardiac death in adults late after repair of tetralogy of Fallot, J. Am. Coll. Cardiol. 40 (2002) 1675-1680.

[23] G.P. Diller, A. Kempny, E. Liodakis, et al., Left ventricular longitudinal function predicts life-threatening ventricular arrhythmia and death in adults with repaired tetralogy of fallot, Circulation 125 (2012) 2440-2446.

[24] Y.F. Cheung, S.J. Wong, X.C. Liang, E.W. Cheung, Torsional mechanics of the left ventricle in patients after surgical repair of tetralogy of Fallot, Circ. J. 75 (2011) 1735-1741.

[25] M.E. Menting, J.A. Eindhoven, A.E. van den Bosch, et al., Abnormal left ventricular rotation and twist in adult patients with corrected tetralogy of Fallot, Eur. Heart J. Cardiovasc. Imaging 15 (2014) 566-574.

[26] F. Haddad, R. Doyle, D.J. Murphy, S.A. Hunt, Right ventricular function in cardiovascular disease, part II: pathophysiology, clinical importance, and management of right ventricular failure, Circulation 117 (2008) 1717-1731.

[27] A. Kempny, G.P. Diller, S. Orwat, et al., Right ventricular-left ventricular interaction in adults with tetralogy of Fallot: a combined cardiac magnetic resonance and echocardiographic speckle tracking study, Int. J. Cardiol. 154 (2012) 259-264.

[28] T. Le Tourneau, D. Messika-Zeitoun, A. Russo, et al., Impact of left atrial volume on clinical outcome in organic mitral regurgitation, J. Am. Coll. Cardiol. 56 (2010) 570-578.

[29] A.N. Borg, K.A. Pearce, S.G. Williams, S.G. Ray, Left atrial function and deformation in chronic primary mitral regurgitation, Eur. J. Echocardiogr. 10 (2009) 833-840.

[30] G.P. Aurigemma, J.S. Gottdiener, A.M. Arnold, M. Chinali, J.C. Hill, D. Kitzman, Left atrial volume and geometry in healthy aging: the Cardiovascular Health Study, Circ. Cardiovasc. Imaging 2 (2009) 282-289.

[31] C. Russo, R.T. Hahn, Z. Jin, S. Homma, R.L. Sacco, M.R. Di Tullio, Comparison of echocardiographic single-plane versus biplane method in the assessment of left atrial volume and validation by real time three-dimensional echocardiography, J. Am. Soc. Echocardiogr. 23 (2010) 954-960. 